Sandra Meinershagen, Department of Mathematics and Statistics, University of Missouri-Kansas City, Kansas City, MO 64110

\title{
THE HAUSDORFF MEASURE AND THE PACKING MEASURE ON A PERTURBED CANTOR SET
}

\begin{abstract}
Baek in [2] and [3] defines the covering measures $h^{s}$ and $Q^{s}$ for a perturbed Cantor set $F$. He shows that when $s$ is the dimension of the covering measures $h^{s}$ and $Q^{s}$ on $F$, then $s$ is the Hausdorff dimension and the packing measure dimension on $F$. In this paper, it is shown that for the perturbed Cantor set $F$, the Hausdorff measure is equal to the covering measure $h^{s}$ on $F$. Under more restrictions on the set $F$, the packing measure is equal to $2 \cdot Q^{s}$. Similar results are shown for the weakly convergent deranged Cantor set.
\end{abstract}

\section{Preliminaries.}

In [3], Baek defined a weakly convergent deranged Cantor set as follows: Let $I=[0,1]$. The left subinterval $I_{\sigma, 1}$ and the right subinterval $I_{\sigma, 2}$ of $I_{\sigma}$ are obtained by deleting the middle open interval of $I_{\sigma}$, labeled $J_{\sigma}$, inductively for each $\sigma \in\{1,2\}^{n}$, where $n=1,2,3, \ldots$. Consider $E_{n}=\cup_{\sigma \in\{1,2\}^{n}} I_{\sigma}$. Then $\left\{E_{n}\right\}$ is a decreasing sequence of closed sets. For each $n$, let $\frac{\left|I_{\sigma, 1}\right|}{\left|I_{\sigma}\right|}=c_{\sigma, 1}$ and $\frac{\left|I_{\sigma, 2}\right|}{\left|I_{\sigma}\right|}=c_{\sigma, 2}$ for all $\sigma \in\{1,2\}^{n}$, where $|I|$ denotes the diameter of $I$. Then $F=\cap_{n=1}^{\infty} E_{n}$ is a weakly convergent deranged Cantor set. Note that if $c_{\sigma, 1}=a_{n+1}$ and $c_{\sigma, 2}=b_{n+1}$ for all $\sigma \in\{1,2\}^{n}$, then $F=\cap_{n=1}^{\infty} E_{n}$ is a perturbed Cantor set as in [2].

The purpose of this paper is to give explicit formulas for the exact value of the Hausdorff measure and for the exact value of the packing measure of $\mathrm{F}$ at

Key Words: Hausdorff measure, packing measure

Mathematical Reviews subject classification: 28A80

Received by the editors January 17, 2001 
the critical dimension. In fact, we prove that if s equals the common value of the Hausdorff dimension and the packing dimension of the perturbed Cantor set $F$ and $\inf _{k}\left\{a_{k}, b_{k}, 1-\left(a_{k}+b_{k}\right)\right\}>0$, then the $s$-dimensional Hausdorff measure, $H^{s}(F)$, of $F$ is given by $H^{s}(F)=\liminf _{n} \prod_{k=1}^{n}\left(a_{k}^{s}+b_{k}^{s}\right)$, and if, in addition, $1-\left(a_{k}+b_{k}\right) \geq \frac{1}{2} \max \left\{a_{k}, b_{k}\right\}$ for all $k$, then the $s$-dimensional packing measure, $p^{s}(F)$, of $F$ is given by $p^{s}(F)=2 \cdot \lim \sup _{n} \prod_{k=1}^{n}\left(a_{k}^{s}+b_{k}^{s}\right)$. We remark that only very few non-trivial examples of sets $F$ for which the exact value of the Hausdorff measure and/or the packing measure of $F$ at the critical dimension are known, cf. the almost forgotten papers by Marion [9], [10] and the more recent papers [1], [6], [8], [16], [17]. Also, Roy Davies has an unpublished proof that the Hausdorff 1-dimensional measure of 1/4 Cantor set in the square is $\sqrt{2}$. The proof of this result of Davies can be found in [17] (The author wishes to thank the referee for the wording of the above paragraph.)

The s-dimensional Hausdorff measure of $F$ is defined by

$$
H^{s}(F)=\lim _{\delta \rightarrow 0} H_{\delta}^{s}(F)
$$

where $H_{\delta}^{s}(F)=\inf \left\{\sum_{n=1}^{\infty}\left|U_{n}\right|^{s}:\left\{U_{n}\right\}_{n=1}^{\infty}\right.$ is a $\delta$-cover of $\left.F\right\}$ and the Hausdorff dimension of $F$ is defined by

$$
\operatorname{dim}_{H}(F)=\sup \left\{s>0: H^{s}(F)=\infty\right\}=\inf \left\{s>0: H^{s}(F)=0\right\} .
$$

The s-dimensional packing measure of $F$ is defined by

$$
p^{s}(F)=\inf \left\{\sum_{n=1}^{\infty} P^{s}\left(F_{n}\right): \cup_{n=1}^{\infty} F_{n}=F\right\},
$$

where $P^{s}(E)=\lim _{\delta \rightarrow 0} P_{\delta}^{s}(E)$ and

$$
P_{\delta}^{s}(E)=\sup \left\{\sum_{n=1}^{\infty}\left|U_{n}\right|^{s}:\left\{U_{n}\right\} \text { is a } \delta \text {-packing of } E\right\} .
$$

The packing dimension of $F$ is

$$
\operatorname{dim}_{P}(F)=\sup \left\{s>0: p^{s}(F)=\infty\right\}=\inf \left\{s>0: p^{s}(F)=0\right\} .
$$

A pseudo-packing of a set $E$ is any family $\mathbf{B}$ of bounded subsets of the real line such that, if $B, B^{\prime} \in \mathbf{B}$, then $B^{c} \cap E^{c} \neq \emptyset$ and $B \cap B^{\prime} \cap E=\emptyset$ where $B^{c}=$ the closure of $B$. A pseudo-packing $\mathbf{B}$ of $E$ by balls centered in $E$ has the 
following property. If $B_{r}(x), B_{s}(y) \in \mathbf{B}$, then $y \notin B_{r}(x)$. A pseudo-packing gives rise to a pre-measure defined as

$$
\begin{array}{r}
R^{s}(E)=\lim _{\delta \rightarrow 0}\left[\operatorname { s u p } \left\{\sum_{n}\left|U_{n}\right|^{s}:\left\{U_{n}\right\} \text { is a } \delta \text {-pseudo-packing of } E\right.\right. \text { by closed } \\
\text { symmetric intervals centred in } E \text { with radii less than } \delta\}]
\end{array}
$$

and the pseudo-packing measure is $r^{s}(E)=\inf \left\{\sum_{k} R^{s}\left(E_{k}\right): E \subset \cup_{k} E_{k}\right\}$. Raymond and Tricot [12] defined the above measure and showed that for any set $F, r^{s}(F)=p^{s}(F)$. [Proposition 10.1]

The $s$-dimensional packing measure is also equal to the following definition of a symmetric derivation basis measure where the positive real function $\delta(x)$ replaces the constant $\delta>0$. A $\delta(\cdot)$-packing of the set $F$ is any disjoint collection of symmetric intervals $B_{r_{i}}\left(x_{i}\right)$ where $x_{i} \in F, r_{i}<\delta\left(x_{i}\right)$, and $P_{\delta(\cdot)}^{s}(F)=\sup \left\{\sum_{i}\left(2 r_{i}\right)^{s}:\left\{B_{r_{i}}\left(x_{i}\right)\right\}\right.$ is a $\delta(\cdot)$-packing of $\left.F\right\}$. The symmetric derivation basis measure is $p^{s}(F)=\inf \left\{P_{\delta(\cdot)}^{s}(F): \delta(\cdot)\right.$ is any positive real function\}. The same notation is being used as for the packing measure because it was shown in [11] that the two measures are equal for all sets $F$.

In [3] for the weakly convergent deranged Cantor set, Baek introduces the covering functions

$$
h^{s}(F)=\liminf _{n \rightarrow \infty} \min _{\sigma \in\{1,2\}^{n}}\left(c_{1}^{s}+c_{2}^{s}\right)\left(c_{\sigma \mid 1,1}^{s}+c_{\sigma \mid 1,2}^{s}\right) \ldots\left(c_{\sigma \mid n, 1}^{s}+c_{\sigma \mid n, 2}^{s}\right)
$$

and

$$
Q^{s}(F)=\limsup _{n \rightarrow \infty} \max _{\sigma \in\{1,2\}^{n}}\left(c_{1}^{s}+c_{2}^{s}\right)\left(c_{\sigma \mid 1,1}^{s}+c_{\sigma \mid 1,2}^{s}\right) \ldots\left(c_{\sigma \mid n, 1}^{s}+c_{\sigma \mid n, 2}^{s}\right) .
$$

For the perturbed Cantor set in [2],

$$
h^{s}(F)=\liminf _{n \rightarrow \infty} \prod_{k=1}^{n}\left(a_{k}^{s}+b_{k}^{s}\right) \text { and } Q^{s}(F)=\limsup _{n \rightarrow \infty} \prod_{k=1}^{n}\left(a_{k}^{s}+b_{k}^{s}\right) .
$$

In [2] and [3], Baek uses $h^{s}(F)$ and $Q^{s}(F)$ to show that if $c_{\sigma, 1}^{s_{\sigma}}+c_{\sigma, 2}^{s_{\sigma}}=1$ (or $a_{k}^{s_{k}}+b_{k}^{s_{k}}=1$ ) and $\lim _{n \rightarrow \infty} s_{\sigma}=s$, for all $\sigma \in\{1,2\}^{n}\left(\right.$ or $\lim _{k \rightarrow \infty} s_{k}=s$ ), then the dimension $h^{s}(F)$ (likewise $Q^{s}(F)$ ) equals $s$ which is the Hausdorff dimension and the packing measure dimension.

\section{Main Results.}

Let $F$ denote a perturbed Cantor set determined by the contracting ratios $a_{n+1}=\frac{\left|I_{\sigma, 1}\right|}{\left|I_{\sigma}\right|}, b_{n+1}=\frac{\left|I_{\sigma, 2}\right|}{\left|I_{\sigma}\right|}$ for all $\sigma \in\{1,2\}^{n}$ and gap ratio $d_{n+1}=1-$ 
$\left(a_{n+1}+b_{n+1}\right)$. Assume that the contracting ratios are uniformly bounded away from 0 in the sense that there exists an $a>0$ such that $a_{n}, b_{n}$ and $d_{n}$ are greater than or equal to $a$ for all $n=1,2, \ldots$. The covering measure $h^{s}$ is defined as

$$
h^{s}(F)=\liminf _{n \rightarrow \infty} \sum_{\sigma \in\{1,2\}^{n}}\left|I_{\sigma}\right|^{s}=\liminf _{n \rightarrow \infty} \prod_{k=1}^{n}\left(a_{k}^{s}+b_{k}^{s}\right) .
$$

The following theorem shows that the covering measure $h^{s}$ is equal to the Hausdorff measure on a perturbed Cantor set.

Theorem 1. Let $F$ be a perturbed Cantor set with contracting ratios $a_{n}$ and $b_{n}$ such that $a_{n}^{s_{n}}+b_{n}^{s_{n}}=1$. Assume that $s_{n} \rightarrow s$ as $n \rightarrow \infty$. Then the Hausdorff measure $H^{s}(F)=h^{s}(F)=\liminf _{n \rightarrow \infty} \prod_{k=1}^{n}\left(a_{k}^{s}+b_{k}^{s}\right)$.

Proof. The dimension of the Hausdorff measure $H^{s}(F)$ and the dimension of the covering measure $h^{s}(F)$ are both equal to $s$ by [2]. Note that $\prod_{k=1}^{n}\left(a_{k}^{s}+b_{k}^{s}\right)=\sum_{\sigma \in\{1,2\}^{n}} I_{\sigma}$. Also note that for each number $n,\left\{I_{\sigma}\right\}_{\sigma \in\{1,2\}^{n}}$ is a covering of $F$. Therefore, $H_{\delta}^{s}(F) \leq \sum_{\sigma \in\{1,2\}^{n}}\left|I_{\sigma}\right|^{s}$ where $\left|I_{\sigma}\right|<\delta$ for all $\sigma \in\{1,2\}^{n}$. Since $\lim _{n \rightarrow \infty}\left|I_{\sigma}\right|=0$ for each $\sigma \in\{1,2\}^{n}$, there exists an $N$ such that if $n \geq N$, then $\left|I_{\sigma}\right|<\delta$ for all $\sigma \in\{1,2\}^{n}$. Therefore, $H_{\delta}^{s}(F) \leq \inf _{n \geq N} \sum_{\sigma \in\{1,2\}^{n}}\left|I_{\sigma}\right|^{s}$ and $H_{\delta}^{s}(F) \leq \liminf _{n \rightarrow \infty} \sum_{\sigma \in\{1,2\}^{n}}\left|I_{\sigma}\right|^{s}$. Hence $H^{s}(F) \leq \liminf _{n \rightarrow \infty} \sum_{\sigma \in\{1,2\}^{n}}\left|I_{\sigma}\right|^{s}$. Let $r>0$ such that $H^{s}(F)<r$. Then $H_{\delta}^{s}(F)<r$ for all $\delta>0$ which implies that there exists a $\delta$-covering $\left\{U_{n}^{\delta}\right\}_{n}$ of $F$ such that $\sum_{n}\left|U_{n}^{\delta}\right|^{s}<r$ for each $\delta>0$. Suppose that there exists a natural number $N$ such that if $n \geq N$, then $\sum_{\sigma \in\{1,2\}^{n}}\left|I_{\sigma}\right|^{s}>r$. Consider $\sum_{\sigma \in\{1,2\}^{N}}\left|I_{\sigma}\right|^{s}$. Let $\delta_{0}=\max \left\{\left|I_{\sigma}\right|: \sigma \in\{1,2\}^{N}\right\}$. Then, $\sum_{n}\left|U_{n}^{\delta_{0}}\right|^{s}<r<$ $\sum_{\sigma \in\{1,2\}^{N}}\left|I_{\sigma}\right|^{s}$. This implies that there exists an $M$ such that $U_{M}^{\delta_{0}} \neq I_{\sigma}$ for all $\sigma \in\{1,2\}^{n}$ and for all $n$. (For, if not, and each $U_{n}^{\delta_{0}}=I_{\sigma}$ where $\sigma \in\{1,2\}^{m}$ for some $m$, then by a finite process described in [7], p. 16, Remark 2.12 which is a method used by A. F. Beardon, On the Hausdorff dimension of the general Cantor set, Proc, Cambridge Phil. Soc., 61 (1965), 679-94 and I. J. Good, The fractal dimension theory of continued fractions, Proc, Cambridge Phil. Soc., 37(1941), 199-288 there would exist an $n$ such that $\sum_{\sigma \in\{1,2\}^{n}}\left|I_{\sigma}\right|^{s}<r$.) (The remainder of this paragraph is referred to as Argument $(\ddagger)$. later in the paper.) Therefore, there exists a $J_{\sigma} \subset I_{\sigma}$ where $\sigma \in\{1,2\}^{N}$ such that $J_{\sigma}$ is not contained in $U_{M}^{\delta_{0}}$; however, $U_{M}^{\delta_{0}} \supset J_{\gamma}$ where $\gamma \in\{1,2\}^{K}, K<N$, and $\left|J_{\sigma}\right|>\left|J_{\gamma}\right|$. Now, $I_{\sigma} \subset I_{\gamma}$ which implies that $\left|I_{\sigma}\right|<\left(\frac{1}{2}\right)^{N-K}\left|I_{\gamma}\right|$. Therefore, 


$$
\begin{aligned}
& \frac{1}{\left|I_{\sigma}\right|}>\frac{2^{N-K}}{\left|I_{\gamma}\right|} \text { which implies that } \\
& \quad d_{N+1}=\frac{\left|J_{\sigma}\right|}{\left|I_{\sigma}\right|}>\frac{2^{N-K}\left|J_{\sigma}\right|}{\left|I_{\gamma}\right|}>\frac{2^{N-K}\left|J_{\gamma}\right|}{\left|I_{\gamma}\right|}=2^{N-K} d_{K+1}>2^{N-K} a
\end{aligned}
$$

where $a>0$ is the uniform lower bound for $a_{k}, b_{k}$, and $d_{k}$ for all $\mathrm{k}$. Consider $\sum_{\sigma \in\{1,2\}^{N+1}}\left|I_{\sigma}\right|^{s}>r$. Again let $\delta_{1}=\max \left\{\left|I_{\sigma}\right|: \sigma \in\{1,2\}^{N+1}\right\}$. Then, there exists a $U_{M_{1}}^{\delta_{1}} \neq I_{\sigma}$ for all $\sigma \in\{1,2\}^{n}$ and for all $n$ which implies that there exists a $\sigma_{1} \in\{1,2\}^{N+1}$ such that $J_{\sigma_{1}} \subset I_{\sigma_{1}}$ and $J_{\sigma_{1}}$ is not contained in $U_{M_{1}}^{\delta_{1}}$; however, $U_{M_{1}}^{\delta_{1}} \supset J_{\gamma_{1}}$ for some $\gamma_{1} \in\{1,2\}^{K_{1}}$ and $K_{1}<N+1$ with $\left|J_{\sigma_{1}}\right|>\left|J_{\gamma_{1}}\right|$. (1) Suppose $K_{1}=N$. Then $\left|J_{\sigma_{1}}\right|>\left|J_{\gamma_{1}}\right|$ implies that $d_{N+2}>2 d_{N+1}>$ $2\left(2^{N-K} a\right) \geq 2^{2} a$. (2) Suppose $K_{1}<N$. Then $d_{N+2}>2^{N+1-K_{1}} d_{K_{1}+1}>$ $2^{N+1-K_{1}} a=2\left(2^{N-K_{1}} a\right) \geq 2^{2} a$. Again, consider $\sum_{\sigma \in\{1,2\}^{N+2}}\left|I_{\sigma}\right|^{s}>r$ and $\delta_{2}=\max \left\{\left|I_{\sigma}\right|: \sigma \in\{1,2\}^{N+2}\right\}$. Then there exists a $U_{M_{2}}^{\delta_{2}} \neq I_{\sigma}$ for all $\sigma \in$ $\{1,2\}^{n}$ and all $n$ which implies there exists a $\sigma_{2} \in\{1,2\}^{N+2}$ such that $J_{\sigma_{2}} \subset$ $I_{\sigma_{2}}, J_{\sigma_{2}} \not \subset U_{M_{2}}^{\delta_{2}}$ but $J_{\gamma_{2}} \subset U_{M_{2}}^{\delta_{2}}$ where $\gamma_{2} \in\{1,2\}^{K_{2}}$ and $K_{2}<N+2$ with $\left|J_{\sigma_{2}}\right|>\left|J_{\gamma_{2}}\right|$. (1) Suppose $K_{2}=N+1$. Then $d_{N+3}>2 d_{N+2}>2\left(2^{2} a\right)=$ $2^{3} a$. (2) Suppose $K_{2}=N$. Then $d_{N+3}>2^{2} d_{N+1}>2^{2}\left(2^{N-K} a\right)>2^{3} a$. (3) Suppose $K_{2}<N$. Then $d_{N+3}>2^{N+3-K_{2}} d_{K_{2}+1}>2^{3} a$. Continuing this process for each natural number $k$ produces a sequence $d_{N+K}>2^{K} a \rightarrow \infty$ as $K \rightarrow \infty$. However, since $a_{k}>a$, and $b_{k}>a$ for all $k, d_{k}=1-\left(a_{k}+b_{k}\right)<1-2 a$ which is a contradiction.

Thus, there exists infinitely many $n$ such that $\sum_{\sigma \in\{1,2\}^{n}}\left|I_{\sigma}\right|^{s}<r$ which implies that $\liminf _{n \rightarrow \infty} \sum_{\sigma \in\{1,2\}^{n}}\left|I_{\sigma}\right|^{s}<r$. Since $r>0$ with $r>H^{s}(F)$ was arbitrary, $\liminf _{n \rightarrow \infty} \sum_{\sigma \in\{1,2\}^{n}}\left|I_{\sigma}\right|^{s} \leq H^{s}(F)$ and, therefore,

$$
H^{s}(F)=\liminf _{n \rightarrow \infty} \sum_{\sigma \in\{1,2\}^{n}}\left|I_{\sigma}\right|^{s}=\liminf _{n \rightarrow \infty} \prod_{k=1}^{n}\left(a_{k}^{s}+b_{k}^{s}\right) .
$$

The following corollary gives the result that the Hausdorff measure is equal to one when the left contracting ratio is equal to $a$ and the right contracting ratio is equal to $b$ for all $k$.

Corollary 2. Let $F$ be a perturbed Cantor set with contracting ratios $a_{k}=a$ and $b_{k}=b$ for all $k$. Let $a^{s}+b^{s}=1$. Then $H^{s}(F)=1$.

Proof. The Hausdorff dimension of $F$ is equal to $s$ by [2]. Also $H^{s}(F)=$ $\liminf _{n \rightarrow \infty} \prod_{k=1}^{n}\left(a_{k}^{s}+b_{k}^{s}\right)$ by Theorem 1. Since $a_{k}=a, b_{k}=b$ and $a^{s}+b^{s}=1$, $H^{s}(F)=1$. 
Now, let $F$ denote a weakly convergent deranged Cantor set determined by the contracting ratios $c_{\sigma}$ and the gap ratios $d_{\sigma}=1-\left(c_{\sigma, 1}+c_{\sigma, 2}\right)$ with $\sigma \in\{1,2\}^{n}$ where $n=1,2, \ldots$. Assume that the $c_{\sigma}$ and $d_{\sigma}$ are locally uniformly bounded away from 0 in the sense that for each $\sigma \in\{1,2\}^{\infty}$, $\left\{c_{\sigma \mid k}\right\}_{k=1}^{\infty}$ and $\left\{d_{\sigma \mid k}\right\}_{k=1}^{\infty}$ are uniformly bounded away from 0 . Note that if $x \in F$, then there is an $\sigma \in\{1,2\}^{\infty}$ such that $\cap_{k=1}^{\infty} I_{\sigma \mid n}=\{x\}$. (Here $\sigma \mid k=\left(i_{1}, i_{2}, \cdots i_{k}\right)$ where $\left.\sigma=\left(i_{1}, i_{2}, \cdots i_{k}, i_{k+1}, \cdots\right)\right)$ Also, assume for the following corollary that for a fixed $N$, the left ratios of each subinterval of $I_{\sigma \mid N}$ are equal to $C_{\sigma \mid N+k, 1}$ meaning that for any $\sigma^{*} \in\{1,2\}^{\infty}$ such that $\sigma^{*}|N=\sigma| N$, $c_{\sigma^{*} \mid N+k, 1}=C_{\sigma \mid N+k, 1}$. Assume similarly for $C_{\sigma \mid N+k, 2}$ in each right ratio of each subinterval of $I_{\sigma \mid N}$.

Corollary 3. Let $F$ be a weakly convergent deranged Cantor set with contracting ratios $c_{\sigma, 1}$ and $c_{\sigma, 2}$ such that $c_{\sigma, 1}^{s_{\sigma}}+c_{\sigma, 2}^{s_{\sigma}}=1$. Assume $\lim _{n \rightarrow \infty} s_{\sigma}=s$ for each $\sigma \in\{1,2\}^{n}$. For any $n$, let $\left\{I_{\sigma}\right\}_{\sigma \in\{1,2\}^{n}}$ be the closed intervals such that $E_{n}=\cup_{\sigma \in\{1,2\}^{n}} I_{\sigma}$ with $F=\cap_{n=1}^{\infty} E_{n}$. Assume there exists an $N$ such that for each interval $I_{\sigma \mid N}, \sigma \in\{1,2\}^{\infty}$, the left ratios for $n \geq N$ equal $C_{\sigma \mid n, 1}$ and the right ratios equal $C_{\sigma \mid n, 2}$. Then

$H^{s}(F)=\sum_{\sigma \in\{1,2\}^{N}} H^{s}\left(F \cap I_{\sigma}\right)=\sum_{\sigma \in\{1,2\}^{N}}\left(\prod_{k=1}^{N} c_{\sigma \mid k}^{s}\right) \liminf _{m \rightarrow \infty} \prod_{k=N}^{N+m}\left(C_{\sigma \mid k, 1}^{s}+C_{\sigma \mid k, 2}^{s}\right)$

where $\left|I_{\sigma}\right|^{s}=\prod_{k=1}^{N}\left(c_{\sigma \mid k}^{s}\right)$ for each $\sigma \in\{1,2\}^{N}$.

Proof. Since the contracting ratios $\left\{c_{\sigma}\right\}_{\sigma \in\{1,2\}^{n}}$ and gap ratios $\left\{d_{\sigma}\right\}_{\sigma \in\{1,2\}^{n}}$ are locally bounded away from 0 , for each $I_{\sigma \mid N}$ with $\sigma \in\{1,2\}^{\infty}$, the contracting ratios $\left\{c_{\sigma \mid k}\right\}_{k=1}^{\infty}$ and gap ratios $\left\{d_{\sigma \mid k}\right\}_{k=1}^{\infty}$ are uniformly bounded away from 0 . Therefore, $\left\{C_{\sigma \mid k, 1}\right\}_{k=N}^{\infty}$ and $\left\{C_{\sigma \mid k, 2}\right\}_{k=N}^{\infty}$ are uniformly bounded away from 0. By Theorem 1, $H^{s}\left(F \cap I_{\sigma}\right)=\liminf _{m \rightarrow \infty} \sum_{\gamma \in\{1,2\}^{m}}\left|I_{\sigma * \gamma}\right|^{s}$ where $\sigma * \gamma$ is the sequence $\sigma \in\{1,2\}^{N}$ followed by the sequence $\gamma \in\{1,2\}^{m}$ such that $\sigma * \gamma \in\{1,2\}^{N+m}$. Note that $\left|I_{\sigma}\right|^{s}=\prod_{k=1}^{N} c_{\sigma \mid k}^{s}, C_{\sigma \mid N, 1}=\frac{\left|I_{\sigma \mid N, 1}\right|}{\left|I_{\sigma \mid N}\right|}$, and $C_{\sigma \mid N, 2}=\frac{\left|I_{\sigma \mid N, 2}\right|}{\left|I_{\sigma \mid N}\right|}$ where $\left|I_{\sigma \mid N}\right|=\left|I_{\sigma}\right|$. Therefore,

$$
H^{s}\left(F \cap I_{\sigma}\right)=\left(\prod_{k=1}^{N} c_{\sigma \mid k}^{s}\right) \liminf _{m \rightarrow \infty} \prod_{k=N}^{N+m}\left(C_{\sigma \mid k, 1}^{s}+C_{\sigma \mid k, 2}^{s}\right) .
$$


Hence,

$$
\begin{aligned}
H^{s}(F) & =\sum_{\sigma \in\{1,2\}^{N}} H^{s}\left(F \cap I_{\sigma}\right)=\sum_{\sigma \in\{1,2\}^{N}} \liminf _{m \rightarrow \infty} \sum_{\gamma \in\{1,2\}^{m}}\left|I_{\sigma * \gamma}\right|^{s} \\
& =\sum_{\sigma \in\{1,2\}^{N}}\left(\prod_{k=1}^{N} c_{\sigma \mid k}^{s}\right) \liminf _{m \rightarrow \infty} \prod_{k=N}^{N+m}\left(C_{\sigma \mid k, 1}^{s}+C_{\sigma \mid k, 2}^{s}\right) .
\end{aligned}
$$

The analogous results for the packing measure is unknown to the author. However, if the gap ratios in the perturbed Cantor set are restricted to being greater than or equal to $\frac{1}{2} \max \left\{a_{k}, b_{k}\right\}$, the following result holds.

Theorem 4. Let $F$ be a perturbed Cantor set with contracting ratios $a_{k}$ and $b_{k}$ such that $a_{k}^{s_{k}}+b_{k}^{s_{k}}=1$. Assume that the gap ratios $d_{k}=1-\left(a_{k}+b_{k}\right)$ satisfies $d_{k} \geq \frac{1}{2} \max \left\{a_{k}, b_{k}\right\}$ and that $\lim _{k \rightarrow \infty} s_{k}=s$. Then

$$
p^{s}(F)=2 \cdot Q^{s}(F)=2 \cdot \limsup _{n \rightarrow \infty} \prod_{k=1}^{n}\left(a_{k}^{s}+b_{k}^{s}\right) .
$$

Proof. From [2] $p^{s}(F)=Q^{s}(F)=s$. Since the pseudo-packing measure $r^{s}(F)$ is equal to the packing measure by [12]-Proposition 10.1], it suffices to show that $r^{s}(F)=2 \cdot Q^{s}(F)$. Also, since $F$ is a closed, bounded set, $P^{s}(F)=p^{s}(F)=r^{s}(F)=R^{s}(F)$. For each $I_{\sigma}$, with $\sigma \in\{1,2\}^{n}$, let $S^{l}\left(I_{\sigma}\right)$ be the symmetric open interval whose center is the left endpoint of $I_{\sigma}$ and whose radius is $\frac{1}{2}\left|I_{\sigma}\right|$ and let $S^{r}\left(I_{\sigma}\right)$ be the symmetric open interval whose center is the right endpoint of $I_{\sigma}$ and whose radius is $\frac{1}{2}\left|I_{\sigma}\right|$. Note that $\left|S^{l}\left(I_{\sigma}\right)\right|^{s}+$ $\left|S^{r}\left(I_{\sigma}\right)\right|^{s}=2 \cdot\left|I_{\sigma}\right|^{s}$ and $\left\{S^{l}\left(I_{\sigma}\right), S^{r}\left(I_{\sigma}\right)\right\}_{\sigma \in\{1,2\}^{n}}$ is a pseudo-packing of $F$ since $d_{k} \geq \frac{1}{2} \max \left\{a_{k}, b_{k}\right\}$ which means that each interval satisfies $\left|J_{\sigma}\right| \geq$ $\frac{1}{2}\left|I_{\sigma, 1}\right|$ and $\left|J_{\sigma}\right| \geq \frac{1}{2}\left|I_{\sigma, 2}\right|$. Therefore, $\sum_{\sigma \in\{1,2\}^{n}}\left[\left|S^{l}\left(I_{\sigma}\right)\right|^{s}+\left|S^{r}\left(I_{\sigma}\right)\right|^{s}\right] \leq$ $R_{\delta}^{s}(F)$ when $\frac{1}{2}\left|I_{\sigma}\right|<\delta$ for every $\sigma \in\{1,2\}^{n}$. Hence,

$$
2 \cdot Q^{s}(F)=\limsup _{n \rightarrow \infty} \sum_{\sigma \in\{1,2\}^{n}} 2 \cdot\left|I_{\sigma}\right|^{s} \leq R_{\delta}^{s}(F)
$$

for any $\delta>0$ which implies that $2 \cdot Q^{s}(F) \leq R^{s}(F)$. Let $r>0$ be a positive real number such that $0<r<R^{s}(F)$ which implies that $r<R_{\delta}^{s}(F)$ for any $\delta>0$. Therefore, there exists a pseudo-packing $\left\{U_{n}^{\delta}\right\}_{n}$ such that $\sum_{n}\left|U_{n}^{\delta}\right|^{s}>r$ for each $\delta>0$. Suppose that there exists a natural number $N$ such that if $n \geq$ $N$. Then $2 \cdot \prod_{k=1}^{n}\left(a_{k}^{s}+b_{k}^{s}\right)=\sum_{\sigma \in\{1,2\}^{n}} 2 \cdot\left|I_{\sigma}\right|^{s}<r$ for each $n \geq N$. Fix $\delta>0$. Since $\sum_{n}\left|U_{n}^{\delta}\right|^{s}>r$, there exists an $M$ such that $U_{M}^{\delta}=\left(x_{M}-r_{M}, x_{M}+r_{M}\right)$ 
where $x_{M} \in F, r_{M}<\delta$ but $x_{M}$ is not an endpoint of any $I_{\sigma}$ where $\sigma \in\{1,2\}^{n}$ and $n \geq N$. (For, if not, and each midpoint of a $U_{n}^{\delta}$ is an endpoint of some $I_{\sigma}$, then by a finite process described in $[7$, p. 16, Remark 2.12] there would exist an $n$ such that $\sum_{\sigma \in\{1,2\}^{n}} 2 \cdot\left|I_{\sigma}\right|^{s}>r$.) Therefore, $x_{M}$ is a limit point of $F$ which implies that there exists a $\sigma \in\{1,2\}^{\infty}$ such that $x_{M} \in I_{\sigma \mid k}$, where $\sigma \mid k \in\{1,2\}^{k}$, for $k=1,2, \ldots$ and $x_{M}=\cap_{k=1}^{\infty} I_{\sigma \mid k}$. Let $N_{0}$ be the first natural number larger than $N$ such that $\frac{1}{2}\left|I_{\sigma}\right|<\delta$ for every $\sigma \in\{1,2\}^{N_{0}}$. Then

$$
r<\sum_{n}\left|U_{n}^{\delta}\right|^{s} \leq\left|U_{M}^{\delta}\right|^{s}+\sum_{\sigma \neq \gamma \in\{1,2\}^{N_{0}}}\left(\left|S^{l}\left(I_{\gamma}\right)\right|^{s}+\left|S^{r}\left(I_{\gamma}\right)\right|^{s}\right) .
$$

However,

$$
\left(\left|S^{l}\left(I_{\sigma \mid N_{0}}\right)\right|^{s}+\left|S^{r}\left(I_{\sigma \mid N_{0}}\right)\right|^{s}\right)+\sum_{\sigma \mid N_{0} \neq \gamma \in\{1,2\}^{N_{0}}}\left[\left|S^{l}\left(I_{\gamma}\right)\right|^{s}+\left|S^{r}\left(I_{\sigma}\right)\right|^{s}\right]<r
$$

where $\sigma \mid N_{0} \in\{1,2\}^{N_{0}}$. This implies that

$$
2 \cdot\left|I_{\sigma \mid N_{0}}\right|^{s}=\left|S^{l}\left(I_{\sigma \mid N_{0}}\right)\right|^{s}+\left|S^{r}\left(I_{\sigma \mid N_{0}}\right)\right|^{s}<\left|U_{M}^{\delta}\right|^{s}=\left(2 r_{M}\right)^{s}
$$

which means that $r_{M}>\left|I_{\sigma \mid N_{0}}\right|$. Without loss of generality, assume that $I_{\sigma \mid N_{0}}=I_{\sigma \mid\left(N_{0}-1\right), 1}$ and that $\frac{\left|I_{\sigma \mid N_{0}}\right|}{\left|I_{\sigma \mid N_{0}-1}\right|}=a_{N_{0}}$. In order for the inequality (1) to hold with $s<1$, the gap interval $J_{\sigma \mid N_{0}-1}$ in the interval $I_{\sigma \mid N_{0}-1}$ (where $d_{N_{0}}=\frac{\left|J_{\sigma \mid N_{0}-1}\right|}{\left|I_{\sigma \mid N_{0}-1}\right|}$ ) must be very large and $\left|I_{\sigma \mid N_{0}}\right|$ must be very small. However, by Argument $(\ddagger)$ in Theorem $1, J_{\sigma \mid N_{0}-1}$ cannot by disproportionately large. Therefore, $J_{\sigma \mid N_{0}-1}$ must be very off center and $I_{\sigma \mid N_{0}}$ is very small which implies that $a_{N_{0}}=r_{N_{0}} b_{N_{0}}$ where $r_{N_{0}}<1$. Again, let $\delta_{1}=$ $\max \left\{\frac{1}{2}\left|I_{\sigma}\right|: \sigma \in\{1,2\}^{N_{0}+1}\right\}$. Then, there exists a packing $\left\{U_{n}^{\delta_{1}}\right\}$ such that $r<\sum_{n}\left|U_{n}^{\delta_{1}}\right|^{s}$ and $\sum_{\sigma \in\{1,2\}^{N_{0}+1}}\left[\left|S^{l}\left(I_{\sigma}\right)\right|^{s}+\left|S^{r}\left(I_{\sigma}\right)\right|^{s}\right]<r$. Therefore, again, there exists an $M_{1}$ such that $U_{M_{1}}^{\delta_{1}}=\left(x_{M_{1}}-r_{M_{1}}, x_{M_{1}}+r_{M_{1}}\right)$ where $x_{M_{1}} \in F$, $r_{M_{1}}<\delta_{1}$ and $x_{M_{1}}$ is not an endpoint of any $I_{\sigma}, \sigma \in\{1,2\}^{n}$ and $n \geq N$. Likewise, there exists a $\sigma_{1} \in\{1,2\}^{\infty}$ such that $\left\{x_{M_{1}}\right\}=\cap_{k=1}^{\infty} I_{\sigma_{1} \mid k}$ and $\left|S^{l}\left(I_{\sigma_{1} \mid N_{0}+1}\right)\right|^{s}+\left|S^{r}\left(I_{\sigma_{1} \mid N_{0}+1}\right)\right|^{s}<\left|U_{M_{1}}^{\delta_{1}}\right|^{s}$ which implies that $r_{M_{1}}>\left|I_{\sigma_{1} \mid N_{0}+1}\right|$ and $a_{N_{0}+1}=r_{N_{0}+1} b_{N_{0}+1}$ where $r_{N_{0}+1}<1$. Continuing this process for each natural number $k$ produces the equation for the interval $I_{\sigma \mid N_{0}}$

$$
\left|I_{\sigma \mid N_{0}+k, 1}\right|=\prod_{j=0}^{k+1}\left(r_{N_{0}+j} b_{N_{0}+j}\right)\left|I_{\sigma \mid N_{0}-1}\right|
$$


which implies that $a_{N_{0}+k}=\left(\prod_{j=0}^{k} r_{N_{0}+j}\right) b_{N_{0}+k}<\left(\prod_{j=0}^{k} r_{N_{0}+j}\right) \frac{1}{2}$. Since each $J_{\sigma \mid N_{0}+k}$ must be significantly off center, there exists an $\varepsilon>0$ such that $r_{N_{0}+k}<1-\varepsilon$ for all $k$. Therefore,

$$
0<a<a_{N_{0}+k}<\left(\prod_{j=0}^{k} r_{N_{0}+j}\right) \frac{1}{2}<(1-\varepsilon)^{k} \frac{1}{2} \rightarrow 0 \text { as } k \rightarrow \infty
$$

which is a contradiction. Hence, there exists infinitely many $n$ such that $\sum_{\sigma \in\{1,2\}^{n}}\left(\left|S^{l}\left(I_{\sigma}\right)\right|^{s}+\left|S^{r}\left(I_{\sigma}\right)\right|^{s}\right)>r$ which implies that

$$
\limsup _{n \rightarrow \infty} \sum_{\sigma \in\{1,2\}^{n}} 2 \cdot\left|I_{\sigma}\right|^{s} \geq r \text { and } 2 \cdot \limsup _{n \rightarrow \infty} \prod_{k=1}^{n}\left(a_{k}^{s}+b_{k}^{s}\right) \geq r .
$$

Since $r>0$ was arbitrary, $\lim \sup _{n \rightarrow \infty} \sum_{\sigma \in\{1,2\}^{n}} 2\left|I_{\sigma}\right|^{s} \geq R^{s}(F)$ which implies that

$$
2 \cdot \limsup _{n \rightarrow \infty} \prod_{k=1}^{n}\left(a_{k}^{s}+b_{k}^{s}\right)=R^{s}(F)
$$

The following corollary shows that under the restriction that the gap ratio $d \geq \frac{1}{2} \max \{a, b\}$ when $a$ is the left contracting ratio for the simple perturbed Cantor set and $b$ is the right contracting ratio, then the packing measure is 2 .

Corollary 5. Let $F$ be the perturbed Cantor set with contracting ratios $a_{k}=a$ and $b_{k}=b$ for all $k$. Assume that the gap ratio $d=1-(a+b)$ satisfies $d \geq \frac{1}{2} \max \{a, b\}$ and $a^{s}+b^{s}=1$.. Then $P^{s}(F)=2$.

Proof. By [2], $s$ is the packing measure dimension of $F$. By Theorem 4, $p^{s}(F)=2 \limsup _{n \rightarrow \infty} \prod_{k=1}^{n}\left(a_{k}^{s}+b_{k}^{s}\right)=2 \limsup _{n \rightarrow \infty} \prod_{k=1}^{n}\left(a^{s}+b^{s}\right)=2$.

Now for the result analogous to Corollary 3 for the more general weakly convergent deranged Cantor set $F$.

Corollary 6. Let $F$ be a weakly convergent deranged Cantor set with contracting ratios $c_{\sigma, 1}$ and $c_{\sigma, 2}$ such that $c_{\sigma, 1}^{s_{\sigma}}+c_{\sigma, 2}^{s_{\sigma}}=1$. Assume that the gap ratios $d_{\sigma}=1-\left(c_{\sigma, 1}+c_{\sigma, 2}\right)$ satisfy $d_{\sigma} \geq \frac{1}{2} \max \left\{c_{\sigma, 1}, c_{\sigma, 2}\right\}$, and there exists an $N$ such that for each interval $I_{\sigma \mid N}, \sigma \in\{1,2\}^{\infty}$, the left ratios of each left subinterval of $I_{\sigma \mid N}$ equal $C_{\sigma \mid n, 1}$ and the right ratios equal $C_{\sigma \mid n, 2}$ for $n \geq N$. 
Assume also that $\lim _{n \rightarrow \infty} s_{\sigma}=s$ for each $\sigma \in\{1,2\}^{n}$. Then

$$
\begin{aligned}
p^{s}(F) & =\sum_{\sigma \in\{1,2\}^{N}} p^{s}\left(F \cap I_{\sigma}\right) \\
& =\sum_{\sigma \in\{1,2\}^{N}}\left(\prod_{k=1}^{N} c_{\sigma \mid k}^{s}\right) 2 \cdot \limsup _{m \rightarrow \infty} \prod_{k=N}^{N+m}\left(C_{\sigma \mid k, 1}^{s}+C_{\sigma \mid k, 2}^{s}\right) .
\end{aligned}
$$

Proof. The packing measure dimension of $F$ is $s$ by [3]. Let $\left\{I_{\sigma}\right\}_{\sigma \in\{1,2\}^{N}}$ be the set of closed intervals such that $E_{N}=\cup_{\sigma \in\{1,2\}^{N}} I_{\sigma}$ where the left ratios for each left subinterval of $I_{\sigma}$ equal $C_{\sigma \mid n, 1}$ and the right ratios equal $C_{\sigma \mid n, 2}$ for $n \geq N$. Then, the $C_{\sigma \mid n, 1}, C_{\sigma \mid n, 2}$ and $D_{\sigma \mid n}$ are uniformly bounded. By Theorem $4, p^{s}\left(F \cap I_{\sigma}\right)=\left(\prod_{k=1}^{N} c_{\sigma \mid k}^{s}\right) \lim \sup _{m \rightarrow \infty} 2 \cdot \prod_{k=N}^{N+m}\left(C_{\sigma \mid k, 1}^{s}+C_{\sigma \mid k, 2}^{s}\right)$. Therefore, since $\left\{I_{\sigma}\right\}_{\sigma \in\{1,2\}^{N}}$ are pairwise disjoint closed intervals,

$$
\begin{aligned}
p^{s}(F) & =\sum_{\sigma \in\{1,2\}^{N}} p^{s}\left(F \cap I_{\sigma}\right) \\
& =\sum_{\sigma \in\{1,2\}^{N}}\left(\prod_{k=1}^{N} c_{\sigma \mid k}^{s}\right) 2 \cdot \limsup _{m \rightarrow \infty} \prod_{k=N}^{N+m}\left(C_{\sigma \mid k, 1}^{s}+C_{\sigma \mid k, 2}^{s}\right) .
\end{aligned}
$$

Returning now to the perturbed Cantor set $F$ where the gap ratios have no restrictions other than the uniform lower bound $a>0$ which is the same as the lower bound for the left contracting ratios $a_{k}$ and the right contracting ratios $b_{k}$, the following lower bounds and upper bounds hold for the $s$-dimensional packing measure.

Theorem 7. Let $F$ be a perturbed Cantor set with contracting ratios $a_{k}$ and $b_{k}$ such that $a_{k}^{s_{k}}+b_{k}^{s_{k}}=1$. Then

$$
2^{s \cdot} \cdot \liminf _{n \rightarrow \infty} \prod_{k=1}^{n}\left(a_{k}^{s}+b_{k}^{s}\right) \leq p^{s}(F) \leq\left[\frac{2(1-2 a)+1}{a}\right]^{s} \limsup _{n \rightarrow \infty} \prod_{k=1}^{n}\left(a_{k}^{s}+b_{k}^{s}\right) .
$$

Proof. Let $B_{r}(x)$ be any symmetric interval where $x$ is an endpoint of a $I_{\sigma}$ for any $\sigma \in\{1,2\}^{n}, n=1,2, \ldots$ and $r>0$. Let $\mathbf{B}$ be the collection of all $B_{r}(x)$ such that $\frac{a}{2(1-2 a)+1} \leq \frac{\operatorname{diam}\left(B_{r}(x) \cap F\right)}{\operatorname{diam} B_{r}(x)} \leq \frac{1}{2}$ where $d_{k} \leq 1-2 a$ for all $k$ and $0<a<a_{k}, b_{k}$, and $d_{k}$ for all $k$. Let $y \in F$ be any point that is not an endpoint of any $I_{\sigma}, \sigma \in\{1,2\}^{n}$ and $n=1,2, \ldots$. Then, there exists a sequence 
of positive numbers $\left\{t_{i}(y)\right\}_{i}$, depending of $y$, such that $\lim _{i \rightarrow \infty} t_{i}(y)=0$

$$
0<\frac{a}{[2(1-2 a)+1]}-\varepsilon \leq \frac{\operatorname{diam}\left(B_{t_{i}(y)}(y) \cap F\right)}{\operatorname{diam} B_{t_{i}(y)}(y)} \leq \frac{1}{2}+\varepsilon<1
$$

where $0<\varepsilon<\min \left\{\frac{1}{2}, \frac{a}{[2(1-2 a)+1]}\right\}$. Therefore,

$$
\begin{gathered}
\mathbf{B}^{0}=\mathbf{B} \cup\left\{B_{t_{i}(y)}(y): y \in F \text { and } y\right. \text { is not an endpoint of any } \\
\left.I_{\sigma} \text { for } \sigma \in\{1,2\}^{n}, n=1,2, \ldots\right\}
\end{gathered}
$$

is a Vitali covering of $F$ which implies that there exists a disjoint collection of $\left\{B_{r_{i}}\left(x_{i}\right)\right\}_{i} \subset \mathbf{B}^{0}$ such that $p^{s}\left(F \backslash \cup_{i} B_{r_{i}}\left(x_{i}\right)\right)=0$ and $H^{s}\left(F \backslash \cup_{i} B_{r_{i}}\left(x_{i}\right)\right)=$ 0 . The inequality $\operatorname{diam}\left(B_{r_{i}}\left(x_{i}\right) \cap F\right) \leq\left(\frac{1}{2}+\varepsilon\right) \operatorname{diam} B_{r_{i}}\left(x_{i}\right)$ implies that

$$
\left(\frac{1}{2}+\varepsilon\right)^{-s}\left(\operatorname{diam}\left(B_{r_{i}}\left(x_{i}\right) \cap F\right)\right)^{s} \leq\left(2 r_{i}\right)^{s}
$$

and consequently that

$$
\left(\frac{1}{2}+\varepsilon\right)^{-s} H^{s}\left(B_{r_{i}}\left(x_{i}\right) \cap F\right) \leq\left(\frac{1}{2}+\varepsilon\right)^{-s}\left(\operatorname{diam}\left(B_{r_{i}}\left(x_{i}\right) \cap F\right)\right)^{s} \leq\left(2 r_{i}\right)^{s}
$$

for all $i=1,2, \ldots$. Hence,

$$
\begin{aligned}
\left(\frac{1}{2}+\varepsilon\right)^{-s} H^{s}(F) & =\left(\frac{1}{2}+\varepsilon\right)^{-s} \sum_{i} H^{s}\left(B_{r_{i}}\left(x_{i}\right) \cap F\right) \\
& \leq\left(\frac{1}{2}+\varepsilon\right)^{-s} \sum_{i}\left[\operatorname{diam}\left(B_{r_{i}}\left(x_{i}\right) \cap F\right)\right]^{s} \\
& \leq \sum_{i}\left(2 r_{i}\right)^{s} \leq P_{\delta}^{s}(F)
\end{aligned}
$$

where $r_{i} \leq \delta$ for all $i=1,2, \ldots$. Now, let $\delta(x)>0$ be any positive real function and restrict the radii in $\mathbf{B}^{0}$ to those where $r_{i}<\delta\left(x_{i}\right)$. Then, again, a Vitali covering occurs and the inequality (2) is true for any positive function $\delta(x)$. Hence, $\left(\frac{1}{2}+\varepsilon\right)^{-s} H^{s}(F) \leq p^{s}(F)$.

Let $\varepsilon_{0}>0$ be any positive number, and let $\delta(x)$ be any positive real function such that $P_{\delta(\cdot)}^{s}(F)<p^{s}(F)+\varepsilon_{0}$. Then there exists a $\delta(\cdot)$-packing $\left\{U_{n}\right\}_{n}$ such that $p^{s}(F) \leq \sum_{n}\left|U_{n}\right|^{s} \leq p^{s}(F)+\varepsilon_{0}$. Suppose that there exists a $U_{M}$ such that

$$
\frac{a}{2(1-2 a)+1}-\varepsilon>\frac{\operatorname{diam}\left(U_{M} \cap F\right)}{\operatorname{diam} U_{M}}
$$


where $U_{M}=\left(x_{M}-r_{M}, x_{M}+r_{M}\right)$. Since $\frac{a}{2(1-2 a)+1}-\varepsilon<\frac{1}{2}$, we have $\operatorname{diam}\left(U_{M} \cap F\right)<\frac{1}{2} \operatorname{diam} U_{M}$. Also, since (3) holds, $\operatorname{diam}\left(U_{M} \cap F\right)$ is very small and $x_{M}$ must be an endpoint of an $I_{\sigma}$ for some $n$ and some $\sigma \in\{1,2\}^{n}$. Likewise, the gap intervals on both sides of $I_{\sigma}$ must be very large, one of which must be $J_{\sigma \mid n-1}$. Because of argument ( $\left.\ddagger\right)$ in Theorem 1, assume $J_{\sigma \mid n-1}$ is not the gap interval with endpoint $x_{M}$ and that $r_{M} \leq\left|J_{\sigma \mid n-1}\right|+\left|I_{\sigma}\right|$. Then, $\frac{r_{M}}{\left|I_{\sigma \mid n-1}\right|} \leq \frac{\left|J_{\sigma \mid n-1}\right|}{\left|I_{\sigma \mid n-1}\right|}+\frac{\left|I_{\sigma}\right|}{\left|I_{\sigma \mid n-1}\right|} \leq d_{k}+a_{k} \leq(1-2 a)+\frac{1}{2}$ (similarly, for $d_{k}+b_{k}$ ). Hence,

$$
\begin{aligned}
\frac{\operatorname{diam}\left(U_{M} \cap F\right)}{\operatorname{diam} U_{M}} & =\frac{\operatorname{diam}\left(U_{M} \cap F\right)}{2 r_{M}} \geq \frac{\left|I_{\sigma}\right|}{2\left(\left|J_{\sigma \mid n-1}\right|+\mid I_{\sigma}\right)} \\
& \geq \frac{\left|I_{\sigma}\right|}{\left|I_{\sigma \mid n-1}\right|} \cdot \frac{\left|I_{\sigma \mid n-1}\right|}{\left[2\left(\left|J_{\sigma \mid n-1}\right|+\left|I_{\sigma}\right|\right)\right]} \geq a_{k} \cdot \frac{1}{2\left(d_{k}+a_{k}\right)} \\
& \geq \frac{a}{2(1-2 a)+2 \frac{1}{2}}=\frac{a}{2(1-2 a)+1}
\end{aligned}
$$

(where the same inequalities are true for $b_{k}$ ) which is a contridiction to (3). This implies that the $\delta(\cdot)$-packing $\left\{U_{n}\right\}_{n}$ is contained in $\mathbf{B}^{0}$. Therefore, $\left[\frac{a}{2(1-2 a)+1}-\varepsilon\right]<\frac{\operatorname{diam}\left(U_{n} \cap F\right)}{\operatorname{diam} U_{n}}$ or $\left[\frac{a}{2(1-2 a)+1}-\varepsilon\right] \operatorname{diam} U_{n}<\operatorname{diam}\left(U_{n} \cap F\right)$ which implies $\left[\frac{a}{2(1-2 a)+1}-\varepsilon\right]^{s}\left(2 r_{n}\right)^{s}<\operatorname{diam}\left(U_{n} \cap F\right)^{s}$. Therefore,

$$
\begin{aligned}
{\left[\frac{a}{2(1-2 a)+1}-\varepsilon\right]^{s} p^{s}(F) } & \leq\left[\frac{a}{2(1-2 a)+1}-\varepsilon\right]^{s} \sum_{n}\left(2 r_{n}\right)^{s} \\
& <\sum_{n} \operatorname{diam}\left(U_{n} \cap F\right)^{s}<\sum_{n}\left|I_{\sigma_{n}}\right|^{s}
\end{aligned}
$$

where $\left\{I_{\sigma_{n}}\right\}_{n}$ are disjoint and $U_{n} \cap F \subset I_{\sigma_{n}}$ for each $n$. However, $\sum_{n}\left|I_{\sigma_{n}}\right|^{s}$ can be replaced by $\sum_{\sigma \in\{1,2\}^{N}}\left|I_{\sigma}\right|^{s}$ for some $N$ by the finite process described in [7]. Hence, $\left(\frac{a}{2(1-2 a)+1}-\varepsilon\right)^{s} p^{s}(F) \leq \sup _{n \geq N} \sum_{\sigma \in\{1,2\}^{n}}\left|I_{\sigma}\right|^{s}$. Since $\varepsilon_{0}$ was arbitrary, $\left(\frac{a}{2(1-2 a)+1}-\varepsilon\right)^{s} p^{s}(F) \leq \lim \sup _{n \rightarrow \infty} \sum_{\sigma \in\{1,2\}^{n}}\left|I_{\sigma}\right|$ which implies that

$$
p^{s}(F) \leq\left[\frac{a}{2(1-2 a)+1}-\varepsilon\right]^{-s} \limsup _{n \rightarrow \infty} \prod_{k=1}^{n}\left(a_{k}^{s}+b_{k}^{s}\right) .
$$


Since $\varepsilon$ was arbitrary,

$$
2^{s} \liminf _{n \rightarrow \infty} \prod_{k=1}^{n}\left(a_{k}^{s}+b_{k}^{s}\right) \leq p^{s}(F) \leq\left[\frac{2(1-2 a)+1}{a}\right]^{s} \limsup _{n \rightarrow \infty} \prod_{k=1}^{n}\left(a_{k}^{s}+b_{k}^{s}\right)
$$

for any perturbed Cantor set $F$.

For the simple perturbed Cantor set with left contracting ratio equal to $a$ and right contracting ratio equal to $b$, the packing measure has the following upper and lower bounds.

Corollary 8. Let $F$ be the perturbed Cantor set with contracting ratios $a_{k}=a$ and $b_{k}=b$ for all $k$. Assume that $a^{s}+b^{s}=1$. Then $2^{s} \leq p^{s}(F) \leq\left(\frac{2(1-2 c)+1}{c}\right)^{s}$ where $c=\min \{a, b\}$.

For the weakly convergent deranged Cantor set, the packing measure has the following upper and lower bounds when $a_{\sigma \mid N}$ is defined to be the uniform lower bound for all the contracting ratios of all the left subinterval, right subinterval and gap subintervals of $I_{\sigma}$, where $\sigma \in\{1,2\}^{\infty}$.

Corollary 9. Let $F$ be a weakly convergent deranged Cantor set with contracting ratios $c_{\sigma, 1}$ and $c_{\sigma, 2}$ such that $c_{\sigma, 1}^{s_{\sigma}}+c_{\sigma, 2}^{s_{\sigma}}=1$. Assume $\lim _{n \rightarrow \infty} s_{\sigma}=s$ for each $\sigma \in\{1,2\}^{n}$ and that there exists an $N$ such that for each interval $I_{\sigma \mid N}, \sigma \in\{1,2\}^{\infty}$, the left ratio of each left subinterval of $I_{\sigma \mid N}$ is equal to $C_{\sigma \mid n, 1}$ and the right ratios equal $C_{\sigma \mid n, 2}$ for $n \geq N$. Let $a_{\sigma \mid N}$ be the uniform lower bound for $I_{\sigma \mid N}, \sigma \in\{1,2\}^{\infty}$. Then

$$
\begin{aligned}
& 2^{s} \cdot \sum_{\sigma \in\{1,2\}^{N}}\left(\prod_{k=1}^{N} c_{\sigma \mid k}^{s}\right) \liminf _{m \rightarrow \infty} \prod_{k=N}^{N+m}\left(C_{\sigma \mid k, 1}^{s}+C_{\sigma \mid k, 2}^{s}\right) \leq p^{s}(F) \\
& \leq \sum_{\sigma \in\{1,2\}^{N}}\left(\prod_{k=1}^{N} c_{\sigma \mid k}^{s}\right)\left[\frac{2\left(1-2 a_{\sigma \mid N}\right)+1}{a_{\sigma \mid N}}\right]^{s} \limsup _{m \rightarrow \infty} \prod_{k=N}^{N+m}\left(C_{\sigma \mid k, 1}^{s}+C_{\sigma \mid k, 2}^{s}\right) .
\end{aligned}
$$

\section{References}

[1] E. Ayer and R. Strichartz, Exact Hausdorff measure and intervals of maximum densiry for Cantor sets, Trans, Amer. Math, Soc., 351 (1999), $3725-3741$.

[2] In Soo Baek, Dimensions of the perturbed Cantor set, Real Analysis Exchange, 19, (1993-94), 269-273. 
[3] In Soo Baek, Dimensions of weakly convergent deranged Cantor sets, Real Analysis Exchange, 23(1997-98), 689-696.

[4] Gerald A. Edgar, Measure, Topology and Fractal Geometry, SpringerVerlag, 1990.

[5] Kenneth Falconer, Fractal Geometry, Wiley, 1990.

[6] S. M. Jung, On the Hausdorff measure of a class of self-similar sets, Real. Anal. Exchange 24 (1998/99), 1221-137.

[7] Judit Kardos, Measure and Porosity of Subsets of the real line, Dissertation, 1997, University of Missouri, Kansas City, MO.

[8] H. Lee and I. S. Baek, Hausdorff measure of the scattered Cantor sets Kyungpook Math. J.,35 (1996), 687-693.

[9] J. Marion, Mesure de Hausdorff d'un fractal a similitude interne, Amm. Sci. Math. Quebec, 10 (1986), 51-84.

[10] J. Marion, Mesures de Hausdorff d'ensembles fractals, Ann. Sci. Math. Quebec, 11 (1987), 111-132.

[11] Sandra Meinershagen, The symmetric derivation basis measure and the packing measure, Proc. Amer. Math. Soc., 103 (1988), 813-814.

[12] Xavier Saint Raymond and Claude Tricot, Packing regularity of sets in n-space, Math. Proc. Cambridge. Philos. Soc., 103 (1988), 133-145.

[13] S. J. Taylor and C. Tricot, Packing measure and its evaluation for a brownian path, Trans. Amer. Math. Soc., 288 (1985), 679-699.

[14] S. J. Taylor and C. Tricot, The packing measure of rectifiable subsets of the plane, Math. Proc. Cambridge Philos. Soc. 99 (1986), 285-296.

[15] Claude Tricot, Two definitions of fractional dimension, Math. Proc. Cambridge Philos. Soc., 91 (1982), 57-74.

[16] Z. Zhon and L. Feng, A new estimate of the Hausdorff measure of the Sierpinski Gasket, Nonlinearity 13 (2000), 479-491.

[17] Z. Zhou and M. Wu, The Hausdorff measure of a Sierpinski carpet Sci. China Ser. A, 42 (1999), 673-680. 